\title{
A retrospective cohort study on protective efficacy of multidrug antiretroviral treatment in reduction of HIV transmission through the vertical route in West Bengal, India
}

\author{
Suman Ganguly ${ }^{1}$, Debjit Chakraborty ${ }^{2,3}$, Dipendra N. Goswami ${ }^{4,5}$ \\ ${ }^{1}$ West Bengal State AIDS Prevention and Control Society, West Bengal, India \\ ${ }^{2}$ ICMR - National Centre for Disease Informatics and Research, Bangalore, India \\ ${ }^{3}$ State Surveillance Unit, Integrated Disease Surveillance Programme, West Bengal, India \\ ${ }^{4}$ Department of Community Medicine, Calcutta National Medical College, Kolkata, India \\ ${ }^{5}$ Targeted Intervention, West Bengal State AIDS Prevention and Control Society, India
}

\begin{abstract}
Introduction: Human immunodeficiency virus (HIV) is transmitted vertically from an infected mother to her child. This is a potentially preventable route of HIV transmission and such transmission can be reduced significantly with appropriate prevention of parent-to-child transmission (PPTCT) measures. This study was aimed at determining the protective efficacy of a multidrug, PPTCT regimen, i.e. maternal triple-drug anti-retroviral treatment (ART) coupled with extended nevirapine prophylaxis to the HIV-exposed infant, to reduce the risk of vertical transmission of HIV and also identify associated factors.
\end{abstract}

Material and methods: Three hundred thirty-eight HIV-exposed infants whose polymerase chain reaction (PCR) test results were available and whose mothers received ART during October 2014 to September 2016 were studied retrospectively to determine the HIV transmission rate from mother to child. The incidence rate of HIV transmission was compared with an earlier PPTCT regimen, i.e. single-dose nevirapine mother-infant pair from a historical cohort, and preventive fractions were estimated. Univariate analysis was undertaken to identify factors associated with vertical transmission.

Results: HIV transmission was minimized significantly (26\% increase in preventive fraction) with the multidrug PPTCT regimen as compared to the single-dose mother-infant pair nevirapine prophylaxis. Other factors such as gender of infant, duration of pre-delivery ART exposure, time of HIV detection, mode of delivery, method of infant feeding, duration of nevirapine prophylaxis of the infants, etc., were not significantly associated with risk of vertical transmission when mothers were on ART.

Conclussions: Multidrug ART reduces transmission significantly with insignificant contribution of other factors, and this could pave the way towards elimination of paediatric HIV.

HIV AIDS Rev 2018; 17, 2: 128-133 DOI: https://doi.org/10.5114/hivar.2018.76366

Key words: PPTCT, HIV, nevirapine, multidrug ART, protective efficacy.

Address for correspondence: Debjit Chakraborty, ICMR - National Centre for Disease Informatics and Research, Flat -1, Block D6, Kendriya Vihar, Yelahanka, Kogilu Cross, 560064 Bangalore, India, e-mail: djsmile_1979@yahoo.com
Article history:

Received: 26.06.2017

Received in revised form: 13.01.2018

Accepted: 17.01.2018

Available online: 21.05 .2018
International Journal of HIV-Related Problems

HIV \& AIDS

R e vi e w 


\section{Introduction}

Mother-to-child transmission of human immunodeficiency virus (HIV) infection is potentially preventable. The interventions taken to minimize the risk of transmission are globally termed as prevention of mother-to-child transmission (PMTCT) and in India they are known as prevention of parent-to-child transmission (PPTCT). To achieve this, several kinds of interventions have been tried, tested and implemented with a varying success rate. In India, the major intervention which was adopted earlier was single-dose nevirapine to the delivering positive pregnant woman and it was coupled with single-dose nevirapine suspension to the HIV-exposed infant soon after birth (within 72 hours). This intervention was intended for prevention of intra-partum HIV transmission and was able to reduce the risk of transmission to around $9-12 \%$ as compared to the average risk of transmission of about $30 \%$ without intervention. This programme continued in India until NACP (National AIDS Control Programme) phase III. Under NACP phase IV, a new regimen was introduced to participate in the global pledge to eliminate paediatric HIV, i.e. to reduce the risk of HIV transmission to less than 5\%. The new PPTCT protocol introduced in India was triple-drug anti-retroviral treatment (ART) to all positive pregnant women or lactating mothers irrespective of World Health Organization (WHO) clinical staging, immunological status and gestational age and to administer nevirapine prophylaxis to the HIV-exposed infant regularly from birth to a minimum duration of six weeks. If the duration of exposure to ART for HIV-infected pregnant women was suboptimal (less than 24 weeks) during pregnancy and if exclusive breast feeding was adopted for HIVexposed infants, the nevirapine prophylaxis can be extended up to twelve weeks [1]. This new regimen was intended to reduce HIV transmission during antenatal, intranatal and postnatal periods. This regimen is known as the Option-B+ regimen as per recommendations of the $\mathrm{WHO}$ [2] and it was adopted by the Government of India in December 2013. It was implemented in the state of West Bengal in October 2014.

This study intends to evaluate the protective efficacy of the new PPTCT regimen in preventing parent-to-child transmission of HIV infection. It also aims to compare effects of infant feeding options, modes of delivery undertaken, duration of nevirapine prophylaxis offered to the HIV-exposed infants, duration of maternal exposure to ART during the antenatal period, infant's gender based susceptibility to acquire HIV infection, time of maternal HIV detection, etc.

\section{Material and methods}

Study design: Retrospective record-based cohort study carried out through analysis of secondary data.

Study period: Two years (October 2014 to September 2016).

Study population: HIV-exposed infants who were given extended nevirapine prophylaxis from birth and were tested at least at six week using nucleic acid polymerase chain reaction (PCR) testing during the study period and whose mothers were initiated on ART as per the new guideline. A total number of $338 \mathrm{HIV}$-exposed infants were recruited in this study.

Study tool: A pre-designed tracking sheet to linelist the events of PPTCT interventions and outcomes of each HIV-positive pregnant woman.

Data-source: Secondary data collected as per the designed format from PPTCT linelist registers maintained in Integrated Counselling and Testing Centres (ICTCs).

Operational case definition:

- HIV-exposed infant - an infant born of a HIV-infected woman,

- HIV reactive infant - when a blood sample of an infant shows a reactive result in the dry blood spot technique using the nucleic acid PCR method,

- HIV non-reactive infant - when a blood sample of an infant shows a non-reactive result in the dry blood spot technique using the nucleic acid PCR method.

Exclusion criteria:

- HIV reactive orphan infants,

- HIV-exposed infants with inconclusive PCR result,

- multiple pregnancies.

Sample size: 338 HIV-exposed infants whose PCR test results are available and whose mothers received ART following detection of HIV infection as registered in 252 ICTCs in the state of West Bengal, India during the study period.

Analysis techniques: Relative risk (RR) with 95\% confidence interval (CI) was calculated to compare the rate of vertical transmission among the recipient of the new PPTCT regimen, recipient of mother-infant pair of single-dose nevirapine prophylaxis and the mother-infant pair who did not receive any medical intervention for PPTCT. The last two rates were taken from a historical cohort, i.e. similar representative samples from West Bengal deduced and published earlier by the main authors of this study [3]. Preventive fraction was calculated as (1-RR) and expressed as a percentage. Univariate analysis was undertaken for various contributory factors such as gender of the HIV-exposed infants, mode of HIV-positive delivery (normal and caesarean section), feeding options (exclusive breast feeding and exclusive replacement feeding), time of HIV-positive detection (during ANC, during labour or during breast feeding), known HIV-positive women with new pregnancies vs HIV detection during this pregnancy, duration of nevirapine prophylaxis to the infants, timing of ART exposure of HIV-infected mothers before delivery. Multivariate analysis by binary logistic regression was also carried out.

Analysis tool: The data were analyzed in Statistical Package for Social Science Software (version 17).

Ethical considerations: Shared confidentiality was maintained while collecting and analyzing the data. All HIV screening was done with prior consent of the individual as per the guidelines of the National AIDS Control Organization. Ethical clearance was obtained from the Institutional Ethics Committee of Calcutta National Medical College, West Bengal, India. 


\section{Results}

A total of $338 \mathrm{HIV}$-exposed infants were tested for HIV, of whom 11 were found positive (3.3\%).

Female (52\%) infants exceeded male infants. Normal delivery (65\%) and exclusive breast feeding (64\%) were observed as more frequent practices compared to caesarean section and exclusive replacement feeding counterparts respectively. HIV infection was identified in the mothers mostly (93\%) during antenatal check-up although in $7 \%$ cases it was detected during labour and post-partum. Only $16 \%$ of mothers were known as HIV positive and in the remaining $84 \%$ of cases it was detected during the current pregnancy. Fifty-four percent of HIV-exposed infants received nevirapine for 12 weeks and the remaining $46 \%$ for six weeks only (Table 1 ).

Incidence of HIV in a HIV-exposed child was $27 \%$ in absence of any prophylaxis. The incidence was found to be reduced significantly to $10 \%$ and $3 \%$ respectively when single-dose nevirapine and a multidrug ART regimen (i.e. fixed dose combination of tenofovir $300 \mathrm{mg}$ + lamivudine $300 \mathrm{mg}$ + efavirenz $300 \mathrm{mg}$ ) were administered. Here relative risk (RR) for the no prophylaxis group was considered as the reference (1.00) and significant lowering of RR was observed with the use of single-dose nevirapine $(R R=0.38 ; 0.20-0.70)$ and the multidrug ART regimen $(\mathrm{RR}=0.12 ; 0.06-0.25)$. Hence the preventive fraction increased by $26 \%(88-62=26)$ for the multidrug ART regimen from single-dose nevirapine prophylaxis (Table 2).

Univariate analysis to find out the strength of association with other factors is shown in Table 3. While assessing the gender susceptibility, it was found that the risk of female HIV-exposed infants to contract HIV infection (3.95\%) was higher than that for male counterparts $(2.48 \%)$ but it was not statistically significant. Approximately double the number HIV-exposed infants were put on exclusive breast feeding and the data analysis showed that with the current PPTCT regimen the risk of acquisition of HIV to the HIV-exposed infants did not differ significantly. No significant difference was observed in the HIV positivity rate between HIVexposed infants borne by normal delivery and caesarean section. This study showed no significant difference in risk of contracting HIV infection between infants who received six weeks and twelve weeks of nevirapine prophylaxis. There was no significant difference observed in PPTCT outcomes in regard to time of HIV detection whether pregnant women were detected during the antenatal or intra- or post-natal periods. Similarly, this study did not find any significant difference in PPTCT outcomes between already known
HIV-infected women becoming pregnant and women with HIV infection detected during this pregnancy (Table 3).

Although no major significance was observed in univariate analysis, we also conducted a multivariate analysis by binary logistic regression with all these factors to be further assured. Only ART initiation in the post-natal period of the mother was observed to carry statistically significant risk $[\operatorname{Exp}(B)=10.94$; 95\% CI: 1.66-72.16] of transmission as compared to that in the antenatal period. However, this finding needs to be further validated as the $95 \%$ confidence interval was too wide to have a high precision. No other factor was found to significantly affect the HIV transmission rate when the multidrug regimen was administered to the individuals (Table 4).

\section{Discussion}

This study showed that the present PPTCT regimen with triple-drug ARV prophylaxis to the mother along with ex-

Table 1. Distribution of HIV-exposed infants

\begin{tabular}{|c|c|c|}
\hline Total $(N=338)$ & No. & $\%$ \\
\hline \multicolumn{3}{|l|}{ HIV status of infant } \\
\hline HIV-positive & 11 & 3.3 \\
\hline HIV-negative & 327 & 96.7 \\
\hline \multicolumn{3}{|l|}{ Gender of infant } \\
\hline Male & 161 & 47.6 \\
\hline Female & 177 & 52.4 \\
\hline \multicolumn{3}{|l|}{ Infant initiated with } \\
\hline Exclusive breast feeding & 218 & 64.5 \\
\hline Exclusive replacement feeding & 120 & 35.5 \\
\hline \multicolumn{3}{|l|}{ Mode of delivery } \\
\hline Caesarean section & 117 & 34.6 \\
\hline Normal delivery & 221 & 65.4 \\
\hline \multicolumn{3}{|l|}{ Time of detection of HIV } \\
\hline During antenatal period & 315 & 93.2 \\
\hline Direct in labour and during lactation & 23 & 6.8 \\
\hline \multicolumn{3}{|l|}{ Existence of HIV infection } \\
\hline Detected during this pregnancy & 283 & 83.7 \\
\hline Known positive & 55 & 16.3 \\
\hline \multicolumn{3}{|l|}{ Duration of nevirapine exposure to infant } \\
\hline 6 weeks & 157 & 46.4 \\
\hline 12 weeks & 181 & 53.6 \\
\hline
\end{tabular}

Table 2. Distribution of HIV-exposed infants with respect to different kinds of anti-retroviral treatment regimens and non-recipients

\begin{tabular}{l|c|c|c|c}
\hline Treatment & HIV-positive infants & HIV-negative infants & RR (95\% CI) & $(1-\mathrm{RR}) \%(95 \% \mathrm{CI})$ \\
\hline Multidrug regimen & $11(3.3 \%)$ & $327(96.7 \%)$ & $0.12(0.06-0.25)$ & $88(75-94)$ \\
\hline Single-dose nevirapine & $17(10.1 \%)$ & $151(89.9 \%)$ & $0.38(0.20-0.70)$ & $62(30-80)$ \\
\hline No prophylaxis & $15(26.8 \%)$ & $41(73.2 \%)$ & 1.00 (ref) \\
\hline
\end{tabular}


Table 3. Distribution of HIV-exposed infants with respect to various associated factors

\begin{tabular}{|c|c|c|c|}
\hline Factor & HIV-positive infants & HIV-negative infants & $\mathrm{RR}(95 \% \mathrm{Cl})$ \\
\hline \multicolumn{4}{|l|}{ Gender of infant } \\
\hline Male & $4(2.5 \%)$ & $157(97.5 \%)$ & $0.63(0.19-2.11)$ \\
\hline Female & $7(4.0 \%)$ & $170(96 \%)$ & 1.00 \\
\hline \multicolumn{4}{|l|}{ Infant initiated with } \\
\hline Exclusive breast feeding & $7(3.2 \%)$ & $211(96.8 \%)$ & 1.00 \\
\hline Exclusive replacement feeding & $4(3.3 \%)$ & $116(96.7 \%)$ & $1.04(0.31-3.47)$ \\
\hline \multicolumn{4}{|l|}{ Mode of delivery } \\
\hline Caesarean section & $4(3.4 \%)$ & $113(96.6 \%)$ & $1.08(0.32-3.61)$ \\
\hline Normal delivery & $7(3.2 \%)$ & $214(96.8 \%)$ & 1.00 \\
\hline \multicolumn{4}{|l|}{ Time of detection of HIV } \\
\hline During ANC & $9(2.9 \%)$ & $306(97.1 \%)$ & 1.00 \\
\hline Direct in labour and during lactation & $2(8.7)$ & $21(91.3 \%)$ & $3.04(0.69-13.27)$ \\
\hline \multicolumn{4}{|l|}{ Existence of HIV infection } \\
\hline Detected during this pregnancy & $10(3.5 \%)$ & $273(96.5 \%)$ & $1.94(0.25-14.87)$ \\
\hline Known positive & $1(1.8 \%)$ & $54(98.2 \%)$ & 1.00 \\
\hline \multicolumn{4}{|l|}{ Duration of nevirapine exposure to infant } \\
\hline 6 weeks & $5(3.2 \%)$ & $152(96.8 \%)$ & 1.00 \\
\hline 12 weeks & $6(3.3 \%)$ & $175(96.7 \%)$ & $1.04(0.32-3.34)$ \\
\hline
\end{tabular}

tended nevirapine prophylaxis was more effective (by 26\%) than the earlier regimen, i.e. single-dose nevirapine prophylaxis for the mother-infant pair [3]. Therefore the present regimen might have the potential to eliminate paediatric HIV as the vertical transmission rate came down to less than $5 \%$ despite other contributory factors provided all positive pregnancies could be identified. Although the present study has been conducted in a single state (West Bengal) of India, the standard PMTCT protocol was followed as per option $\mathrm{B}+$ theory of the WHO which is practised across the globe. The contributory factors that were considered are applicable for all HIV-infected pregnancy. Therefore the study might be restricted at the local level but the results may be inferred at least for the Indian population, particularly in view of the paucity of pan-Indian data. The findings of the study will also help in policy making and programme planning at a national level and can be used by other researchers performing a meta-analysis in future.

Efficacy of multidrug ART prophylaxis has been tested across the world in different regions with huge success rate. One sub-Saharan Africa based study also demonstrated that the regimen was very effective in reducing mother-to-child HIV transmission [4]. India-based studies were limited and one such study from South India was conducted with 92 positive pregnant women only [5]. This study showed a transmission rate of $3.3 \%$ with ARV prophylaxis similar (3.25\%) to our study.

This study also recommended that duration of prophylaxis and mode of delivery should be based on time of presentation of the HIV-infected pregnant woman. Our study involved a higher number of positive pregnant women and also showed that there was no significant difference in transmission rate for different modes of delivery conducted once the infected mother was on ART. One Cameroon-based study conducted more than a decade ago used three different types of multidrug regimens and it was found that all these regimens brought down the mother-to-child transmission rate to $6.6 \%$ without any difference in efficacies across the regimens [6]. One review in 2008 emphasized that the use of fully suppressive ART initiated during pregnancy and the breastfeeding period, even in women with no indication of treatment, was a potentially effective PMTCT strategy and this might constitute a very relevant alternative when formula feeding was not judged to be safe or well accepted [7]. It was also found that efficacy of single-dose nevirapine with respect to the PMTCT programme was around $10 \%$ and only linking positive pregnant women with ART services could serve best in a favourable outcome [3]. Another Kenya-based study reiterated the same fact that lack of maternal use of highly active antiretroviral therapy was associated with increased risk of HIV transmission to the child [8]. Regarding efficacy of the Option-B+ regimen, also adopted in India, a study conducted in Malawi demonstrated that there was a significant improvement in transmission rate with increased duration of maternal ART predelivery [9], but our study did not find any relationship between duration of pre-delivery ARV exposure and PPTCT outcome.

Breastfeeding by women infected with HIV was found to be associated with highly significant reductions in mortality 
Table 4. Distribution of HIV-exposed infants with respect to various associated factors on multivariate analysis

\begin{tabular}{|c|c|c|c|c|c|c|c|c|}
\hline \multirow[t]{2}{*}{ Factor } & \multirow[t]{2}{*}{ B } & \multirow[t]{2}{*}{ S.E. } & \multirow[t]{2}{*}{ Wald } & \multirow[t]{2}{*}{ df } & \multirow[t]{2}{*}{ Sig. } & \multirow[t]{2}{*}{$\operatorname{Exp}(B)$} & \multicolumn{2}{|c|}{$95.0 \% \mathrm{Cl}$ for $\operatorname{EXP}(\mathrm{B})$} \\
\hline & & & & & & & Lower & Upper \\
\hline \multicolumn{9}{|l|}{ Type of client } \\
\hline Antenatal period (ref) & & & 6.181 & 2 & 0.045 & & & \\
\hline Direct in labour & 17.877 & $1.077 \mathrm{E} 4$ & 0.000 & 1 & 0.999 & 0.000 & 0.000 & \\
\hline Post-delivery & 2.393 & 0.962 & 6.181 & 1 & 0.013 & 10.943 & 1.659 & 72.165 \\
\hline \multicolumn{9}{|l|}{ Type of case } \\
\hline Known vs. new (ref) & 0.210 & 1.158 & 0.033 & 1 & 0.856 & 1.234 & 0.127 & 11.947 \\
\hline $\begin{array}{l}\text { Duration of anti-retroviral therapy } \\
\text { in antenatal period (weeks) }\end{array}$ & -0.026 & 0.029 & 0.822 & 1 & 0.365 & 0.974 & 0.920 & 1.031 \\
\hline \multicolumn{9}{|l|}{ Type of delivery } \\
\hline Caesarean vs. normal delivery (ref) & -0.220 & 0.671 & 0.107 & 1 & 0.743 & 0.803 & 0.215 & 2.991 \\
\hline $\begin{array}{l}\text { Duration of nevirapine prophylaxis } \\
\text { initiated for infant (in weeks) }\end{array}$ & -0.004 & 0.127 & 0.001 & 1 & 0.977 & 0.996 & 0.776 & 1.279 \\
\hline \multicolumn{9}{|l|}{ Gender of the infant } \\
\hline Male vs. female (ref) & 0.654 & 0.655 & 0.997 & 1 & 0.318 & 1.923 & 0.533 & 6.943 \\
\hline \multicolumn{9}{|l|}{ Type of feeding of infant } \\
\hline Exclusive replacement feeding (ref) & & & 0.189 & 2 & 0.910 & & & \\
\hline Exclusive breast feeding & -0.348 & 0.802 & 0.189 & 1 & 0.664 & 0.706 & 0.147 & 3.398 \\
\hline Mixed feeding & -18.172 & $1.770 \mathrm{E} 4$ & 0.000 & 1 & 0.999 & 0.000 & 0.000 & \\
\hline Constant & -3.205 & 1.764 & 3.302 & 1 & 0.969 & 0.041 & & \\
\hline
\end{tabular}

among their children in sub-Saharan Africa [10]. Our study observed that the infant feeding option did not result in any significant difference in PPTCT outcome when the HIVinfected mothers were already put on ART but one study conducted in Southeast Nigeria demonstrated that breastfeeding reduced the efficacy achieved by the use of ARV drugs [11]. In India exclusive breast feeding until the six month of the HIV-exposed infant is a nationally recommended practice and replacement feeding is only recommended if it is affordable, acceptable, feasible, accessible, sustainable and safe. In a resource-limited country, this kind of policy is well accepted and our study is unique, being able to test various factors which are nonexistent in developed nations. In regards to the benefit of caesarean section in preventing mother-to-child transmission of HIV, a meta-analysis involving 15 prospective cohort studies concluded that after adjustment for receipt of antiretroviral therapy, maternal stage of disease, and infant birth weight, the likelihood of vertical transmission of HIV-1 was decreased by approximately 50\% with elective caesarean section, as compared with other modes of delivery [12]. A similar risk reduction effect of caesarean section was also observed in one randomized clinical trial [13]. Our study did not show any significant difference in HIV transmission rate with respect to modes of delivery.

Female infants might be more susceptible to HIV infection, and this was reported in one study on African infants born of HIV-infected mothers [14]. Our study also observed that the rate of female HIV-exposed infants to acquire HIV infection $(3.95 \%)$ was higher than that of their male counterparts $(2.48 \%)$ but this difference was not statistically significant. Regarding duration of nevirapine prophylaxis to the HIV-exposed infants, the SWEN study observed that a 6-week regimen of daily nevirapine might be associated with a reduction in the risk of HIV transmission at 6 weeks of age, while the lack of a significant reduction in the primary endpoint - risk of HIV transmission at 6 months - suggested that a longer course of daily infant nevirapine to prevent HIV transmission via breast milk might be more effective where access to affordable and safe replacement feeding was not yet available [15]. No study could be found which substantiates the benefits of six-week nevirapine prophylaxis versus twelve-week nevirapine prophylaxis. Our study could not detect any significant difference between the two proposed durations of therapy.

With advanced ARV regimens and effective viral suppression during antepartum and intrapartum periods, the risk of acquisition of HIV for the newborn from a HIV-infected mother may come down to less than $1 \%$ as suggested in the Department of Health and Human Service (DHHS) guideline 2017 [16]. As per European AIDS Clinical Society guideline 9.0 part-II (October 2017), if full plasma viral load suppression can be achieved at least by the third trimester of pregnancy and specifically at the time of delivery, the risk of transmission is 0 to $<0.5 \%$ [17]. In the present study, considering unknown viraemia status, different ethnicity and 
metabolic profile, coverage issues, etc, we have been able to achieve a transmission rate of $3.3 \%$ even in a developing country setting, which is very close to the global standards. Hence this finding may pave the way towards further effective implementation of the PPTCT programme in developing countries towards achievement of above targets and thus elimination of paediatric HIV.

There have been a lot of studies across the world on efficacy of the multidrug ARV regimen in reduction of the vertical transmission rate, but very few studies have been conducted in India; in particular, no such studies could be found from the eastern part of India. Apart from dealing with the PPTCT regimen our study also attempted to identify numerous other probable associated factors which almost all other studies could not describe.

\section{Conclusions}

It is evident from this study that the triple-drug ART regimen is more effective as compared to the earlier regimen of single-dose nevirapine prophylaxis for the PPTCT programme. ART reduces the risk of transmission in such a way that the contribution of other factors remains significantly low.

\section{Limitation}

Routine viral load testing for people living with HIV is not recommended by the Government of India. As per the government programme, routine CD4 testing is still recommended and viral load testing is reserved for those showing signs and symptoms of failure to treatment. Viraemia or viral load testing is definitely a well-recognized marker of maternal immune status which can influence mother-tochild transmission. Since the data on viral load are not routinely generated in India as part of the PPTCT programme, we could not assess any association between PMTCT outcome and viral load of the mother.

\section{Conflict of interest}

The authors declare no potential conflicts of interest with respect to the research, authorship, and/or publication of this article.

\section{References}

1. National PPTCT guideline. Available at: http://naco.gov.in/sites/default/files/National_Guidelines_for_PPTCT.pdf (Accessed: 24 June 2017).

2. New guidance on prevention of mother-to-child transmission of HIV and infant feeding in the context of HIV. Available at: http:// www.who.int/hiv/pub/mtct/PMTCTfactsheet/en/ (Accessed: 21 June 2017).

3. Chakraborty D, Ganguly S, Pramanick S, et al. A Retrospective Cohort Study on Protective Efficacy of Intrapartum Nevirapine Prophylaxis to Prevent Parent to Child Transmission of Human Immunodeficiency Virus in West Bengal. Ann Intern Med Dental Res 2016; 2: CM01-CM06.
4. Chi BH, Stringer JS, Moodley D. Antiretroviral drug regimens to prevent mother-to-child transmission of HIV: a review of scientific, program, and policy advances for sub-Saharan Africa. Curr HIV/ AIDS Rep 2013; 10: 124-133.

5. Karthekeyani V, Alexander G, Solomon E, et al. Prevention of Mother to Child Transmission of HIV: Our Experience in South India. J Obstet Gynecol India 2011; 61: 62-66.

6. Tchendjou P, Same-Ekobo C, Nga A, et al. Effectiveness of Multidrug Antiretroviral Regimens to Prevent Mother-to-Child Transmission of HIV-1 in Routine Public Health Services in Cameroon. PLoS One 2010; 5: e10411.

7. Arrive E, Dabis F. Prophylactic antiretroviral regimens for prevention of mother-to-child transmission of HIV in resource-limited settings. Curr Opin HIV AIDS 2008; 3: 161-165.

8. Nduati EW, Hassan AS, Knight MG, et al. Outcomes of prevention of mother to child transmission of the human immunodeficiency virus-1 in rural Kenya - a cohort study. BMC Public Health 2015; 15: 1008 .

9. Kim MH, Ahmed S, Hosseinipour MD, et al. Brief Report: Impact of Option B+ on the Infant PMTCT Cascade in Lilongwe, Malawi. J Acquir Immune Def Syndr 2015; 70: 99-103.

10. Taha TE, Kumwenda NI, Hoover DR, et al. The impact of breastfeeding on the health of HIV-positive mothers and their children in sub-Saharan Africa. Bull World Health Organization 2006; 84: 546-554.

11. Ikechebelu JI, Ugboaja JO, Kalu SO, et al. The outcome of prevention of mother to child transmission (PMTCT) of HIV infection programme in Nnewi, southeast Nigeria. Nigerian J Med 2011; 20: 421-425.

12. International Perinatal HIV Group. The Mode of Delivery and the Risk of Vertical Transmission of Human Immunodeficiency Virus Type 1 - A Meta-Analysis of 15 Prospective Cohort Studies. New Engl J Med 1999; 340: 977-987.

13. The European Mode of Delivery Collaboration. Elective caesareansection versus vaginal delivery in prevention of vertical HIV-1 transmission: a randomized clinical trial. Lancet 1999; 353: 1035-1039.

14. Taha TE, Nour S, Kumwenda NI, et al. Gender differences in perinatal HIV acquisition among African infants. Pediatrics 2005; 115 : e167-172.

15. Six Week Extended-Dose Nevirapine (SWEN) Study Team. Extendeddose nevirapine to 6 weeks of age for infants to prevent HIV transmission via breastfeeding in Ethiopia, India, and Uganda: an analysis of three randomized controlled trials. Lancet 2008; 372: 300-313.

16. Recommendations for the Use of Antiretroviral Drugs in Pregnant Women with HIV Infection and Interventions to Reduce Perinatal HIV Transmission in the United States. Available at: https://aidsinfo.nih. gov/contentfiles/lvguidelines/PerinatalGL.pdf (Accessed: 11 January 2018).

17. Guidelines version 9.0 European AIDS Clinical Society. Available at: http://www.eacsociety.org/files/guidelines_9.0-english.pdf (Accessed: 11 January 2018). 